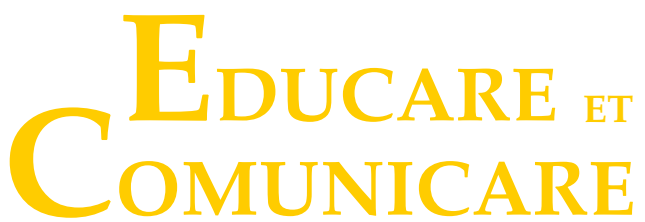

REVISTA CIENTÍFICA DE LA FACULTAD DE HUMANIDADES

https://DOI 10.35383/educare.v9i2.676

e-ISSN: 2312-9018

Universidad Católica Santo Toribio de Mogrovejo

\title{
Eficacia del método del aula invertida en la educación virtual en estudiantes universitarios
}

\author{
Efficacy of the flipped classroom method in virtual education in university students
}

\author{
Walter Hugo Alva Miguel ${ }^{* 1 \text { a }}$ \\ walva@unfv.edu.pe \\ https://orcid.org/0000-0001-9403-4794

\section{César Matos Huamán ${ }^{1 \mathrm{a}}$} \\ cmatos@unfv.edu.pe \\ https://orcid.org/0000-0001-6156-1782

\section{Carlos Zúñiga Reynoso ${ }^{1 \mathrm{a}}$ \\ czuniga@unfv.edu.pe \\ https://orcid.org/0000-0001-6156-1782}

\section{Emma Margarita Wong Fajardo ${ }^{1 \text { a }}$ \\ ewong@unfv.edu.pe \\ https://orcid.org/0000-0002-3775-379X}

\author{
*Autor corresponsal \\ 1 Universidad Nacional Federico Villarreal, Lima, Perú \\ a Doctor
}

\section{Fechas importantes}

Recibido: 07/12/2021

Aceptado: 09/12/2021

Publicado online: $31 / 12 / 2021$

\begin{abstract}
Resumen
El propósito esencial del estudio llevado a cabo fue comprobar objetivamente la eficacia del sistema de aula invertida en los procesos educativos virtuales en la especialidad de Ciencias Histórico-Sociales de la Facultad de Educación de la Universidad Nacional Federico Villarreal En adelante CC. HH.SS. de la UNFV). Con este fin, se desarrolló un estudio de tipo aplicado, descriptivo comparativo, diseño cuasi experimental, de corte longitudinal y con enfoque cuantitativo. Para cumplir esta intención se aplicó un cuestionario previo y posterior a la aplicación del programa, al grupo experimental y al grupo control, con el fin de constatar si se encontraban discrepancias significativas entre dichos grupos al finalizar la experiencia. El instrumento fue validado recurriendo ala opinión de expertos y la confiabilidad comprobada con la prueba Alpha de Cronbach. Este instrumento seadministró a una muestra de34alumnos, 17 de los cuales eran del grupo experimental y los otros 17 al grupo control. Se utilizó el "muestreo no probabilístico" de tipo criterial u opinático para seleccionar el componente muestral. El diseño de investigación asumido fue "cuasi experimental", espeć́ficamente el denominado "diseño antes y después con un grupo control no aleatorizado". El diseño estadístico aplicado fue la "comparación de promedios" utilizándose el programa SPSS versión 22 y el estadístico Razón "t" de Student, para comparar los promedios y verificar las hipótesis. Se constató queel sistema de aula invertida mejora significativamente el desempeño del grupo experimental con referencia al grupo control en el aprendizajedelas asignaturas seleccionadas.
\end{abstract}

Palabras clave: Método del aula invertida, educación virtual, eficacia 


\begin{abstract}
The essential purpose of the study carried out was to objectively verify the effectiveness of the flipped classroom system in virtual educational processes in the specialty of Historical-Social Sciences of the Faculty of Education of the National University Federico Villarreal Hereinafter CC. HH.SS. of the UNFV). To this end, an applied, descriptive comparative study was developed, with a quasi-experimental design, longitudinal section and with a quantitative approach. To fulfill this intention, a questionnaire was applied before and after the application of the program, to the experimental group and the control group, in order to verify if significant discrepancies were found between these groups at the end of the experience. The instrument was validated using the opinion of experts and the reliability verified with the Cronbach's Alpha test. This instrument was administered to a sample of 34 students, 17 of whom were from the experimental group and the other 17 from the control group. Criterial or opinion-type "non-probability sampling" was used to select the sample component. The assumed research design was "quasi-experimental", specifically the so-called "before and after design with a non-randomized control group". The statistical design applied was the "comparison of means" using the SPSS version 22 program and the Student's "t" Ratio statistic, to compare the averages and verify the hypotheses. It was found that the flipped classroom system significantly improves the performance of the Experimental Group with reference to the Control Group in learning the selected subjects.
\end{abstract}

Keywords: Flipped classroom method, virtual education, efficiency.

\title{
Introducción
}

Ante situaciones de crisis nacional e internacional como la generada por la pandemia Covid 19 que ha restringido severamente el desplazamiento físico de las personas y establecido como norma de conducta la "distancia social" con el consiguiente aislamiento social, han aparecido un sinnúmero de soluciones en el campo educativo, uno de los más perjudicados por la emergencia. El campo educativo se ha visto obligado a responder urgentemente ante la emergencia surgida. La Educación Virtual ha sido una de las soluciones más recurridas. Es obvio que existen aspectos de la formación presencial muy difíciles de replicar en la educación online; sin embargo, el principio de realidad está imponiendo la migración a una educación no presencial. Esta situación, inconcebible hasta hace muy poco tiempo, está obligando a los sistemas educativos a plantearse cómo aplicar un nuevo modo de enseñar y aprender. Rodríguez (2019) avizora que para el año 2030, pizarra y plumón, serán obsoletos y sustituidos por laptops y tablets, es decir, el sistema educativo adoptará casi totalmente los medios digitales.

Barrenechea (2020) señala que, en Perú, gran número de infantes y adolescentes están perdiendo la continuidad del periodo escolar por el COVID-19. Muñoz (2020) precisa que los docentes no pueden limitarse a trasladar la metodología presencial a la virtual, ya que es necesario un proceso de adecuación y adaptación, ya que lo contrario sería un grave error. Por tanto, el docente se ve obligado a crear o rediseñar programas virtuales que generen situaciones de aprendizaje adecuadas al nivel de desarrollo personal del estudiante y que tenga muy en cuenta sus necesidades e intereses específicos.

Frente a esta situación se considera, de modo general, que el sistema educativo peruano deberá desarrollar e implementar alternativas educativas digitales considerando que los actuales grupos discentes pertenecen a generaciones nacidas en la era informática.

Los expertos han señalado que el uso de la educación virtual representa numerosas ventajas. Este nuevo entorno permite a los estudiantes adecuarse de manera flexible a los horarios, repasar las clases, mejorar la retroalimentación, desarrollar en la práctica nuevas habilidades y competencias digitales, que ahora son fundamentales para el éxito en su futura vida laboral o profesional.

Puede señalarse que la pandemia ha puesto a prueba a la educación virtual en general y al aprendizaje inverso en particular, como una oportunidad para que el sector educativo continúe operando. La severa crisis desencadenada por la pandemia Covid 19, ha afectado notablemente la continuidad de los estudios universitarios y ha obligado a las instituciones académicas de educación superior a asumir, casi 
obligadamente, nuevas metodologías de aprendizaje, tales como la educación virtual y el aprendizaje inverso, como una forma de afrontar las severas limitaciones académicas impuestas por la pandemia.

El estudio comparó los desempeños de ambos grupos (Experimental y de Control) en las diferentes áreas de la asignatura de Teoría de la Geografía: Unidad I (Evolución histórica de la Geografía), II (Ciencia e investigación científica), III (Geografía científica), IV (Investigación geográfica) y en el promedio general. Se encontraron diferencias significativas entre ambos grupos, las cuales favorecían al Grupo Experimental, diferencias atribuibles al programa aplicado. Las recomendaciones, por tanto, fueron reforzar los procesos educativos virtuales con la modalidad del aprendizaje inverso, como una forma de afrontar las severas limitaciones académicas impuestas por la pandemia.

\section{Problema general}

¿Es posible, que en la Educación Virtual la aplicación del método de aula invertida logre incrementar significativamente el aprendizaje de la asignatura de Teoría de la Geografía en alumnos del tercero y cuarto año de la especialidad de CC.HH. SS, de la Facultad de Educación de la Universidad Nacional Federico Villarreal. 2021?

\section{Problemas específicos}

¿Con la Educación Virtual el método de aula invertida logrará incrementar significativamente el nivel de indicadores de conocimiento en alumnos del tercero y cuarto año de la especialidad de CC.HH.SS, en la Unidad I (Evolución Histórica de la Geografía) de la Facultad de Educación de la UNFV. 2021?

¿Utilizando la Educación Virtual la aplicación del método de aula invertida se podrá incrementar significativamente el nivel de indicadores de conocimiento en alumnos del tercero y cuarto año de la especialidad de CC.HH.SS. en la Unidad II (Ciencia e investigación científica) de la Facultad de Educación de la UNFV. 2021?

¿Aplicando la Educación Virtual con el método de aula invertida se logrará incrementar significativamente el nivel de indicadores de conocimiento en alumnos del tercero y cuarto año de la especialidad de CC.HH.SS. en la Unidad III (Geografía como ciencia) de la Facultad de Educación de la UNFV. 2021?

¿La Educación Virtual con la aplicación del método de aula invertida logrará incrementar significativamente el nivel de indicadores de conocimiento en alumnos del tercero y cuarto año de la especialidad de CC.HH.SS. de la Unidad IV (Investigación geográfica) de la Facultad de Educación de la UNFV. 2021?

\section{Antecedentes}

\section{Nacionales}

Carbajal (2019) analizó con exhaustividad las habilidades profesionales del docente que deben movilizarse en un contexto virtual. Esta investigación partió de los resultados de investigaciones previas con objetivos similares, es decir, que investigaron la eficacia real de estos programas en el nivel de postgrado. Los hallazgos encontrados indican que el sistema virtual requiere complementariamente un docente que apoye de manera idónea el proceso, para lo cual éste debe disponer de una adecuada capacitación pedagógica y tecnológica. Es decir, se requieren docentes digitalmente preparados para asumir adecuadamente las nuevas funciones y capacidades demandadas por la aparición y desarrollo de nuevos procesos educativos de carácter virtual. Tomando en cuenta esta situación, así como sus nuevas demandas y requerimientos, el estudio señalado se centró en las recientes habilidades requeridas del docente, sus nuevos roles y funciones que deben exhibir en los contextos educativos virtuales. Se elaboró y planteó una novedosa propuesta del nuevo perfil profesional del docente virtual, derivada de la aplicación rigurosa de métodos factoriales. 
Domínguez (2019) analizó nuevos desarrollos metodológicos consecuencia del hallazgo de afinidades y convergencia entre las diferentes modalidades educativas, señalando de manera optimista que la tecnología puede favorecer esta integración. Obviamente esta integración implica la eliminación de una serie de modalidades educativas las cuales se hallaban en boga hasta hace poco tiempo, y que se hallaban estructuradas en compartimientos estancos. De esta manera, las diversas investigaciones internacionales están promoviendo la tendencia hacia la integración en una única modalidad educativa, la cual incorporaría en un rubro, las actuales modalidades presencial y virtual. Señala este autor, que esta integración de las modalidades educativas será posible por los avances de las TIC, pudiendo transformarse en una estrategia modelo para que progresivamente se vayan superando los obstáculos e incorporando los beneficios y ventajas de la enseñanza virtual en los procesos de aprendizaje. Las actuales modalidades educativas tienen como objetivo final que los esfuerzos docentes se traduzcan en aprendizajes de los estudiantes. Sin embargo, la educación no puede reducirse a saber transmitir conocimientos. La compleja realidad actual supone la necesidad de desarrollar competencias y aptitudes digitales que es lo que demanda la sociedad actual. El estudio concluyó señalando que la integración de las diversas modalidades educacionales en un solo modelo requiere como complemento que los docentes cuenten con adecuadas competencias digitales. Básicamente, se necesitan docentes expertos y competentes en sus asignaturas, con capacidad para adaptar y aplicar fluidamente sistemas digitales y multimedia en su actividad docente en diversos ambientes educativos.

Cantoni (2018) señaló que la Educación a Distancia, en nuestro país, se encuadra en el Art. 27 de la Ley N. ${ }^{\circ} 28044$, que la define como una modalidad educativa, diferenciada por la interactividad, sincrónica o retrasmitida entre los participantes de la interacción educativa, promovida por procedimientos tecnológicos que respaldan la autonomía del aprendizaje y que puede aplicarse indiscriminadamente a todas las fases del proceso educativo, siempre que se respete la normatividad vigente del caso. Esta autora precisa que la educación a distancia (virtual), tiene como fin complementar, apoyar e, incluso, sustituir a la educación presencial, en función de las circunstancias y condiciones del entorno. Se aprecia en este caso que la legislación vigente aún continúa asignándole a la Educación a Distancia un rol secundario, como si no fuera en la actualidad una opción viable e idónea. Sin embargo, si le reconoce su notable aporte a la función democratizadora de todo proceso educativo, ya que posibilita a discentes, que por diversas razones no pueden acceder a estudios presenciales, incorporarse al sistema educativo. No hay que olvidar que, en nuestro país, abundan los alumnos que, por diversas razones, no pueden seguir estudios presenciales. Ante esta condición, la Educación Virtual surge como alternativa para proseguir viablemente con sus procesos educativos, contribuyendo de esta manera a superar asimetrías y las consiguientes inequidades.

\section{Internacionales}

Según Phipps y Merisotis (2009) amplios estudios consultados precisan que los resultados de aprendizaje que se logran aplicando la educación a distancia no difieren significativamente a obtenidos con la aplicación del método tradicional. Especifican que buena cantidad de investigaciones confirman el hecho que el uso de recientes tecnologías educativas no es tan significativo como la naturaleza intrínseca de las tareas, de las condiciones del educando, de la motivación del docente, sus competencias académicas y metodológicas, la solidez de la capacitación académica del profesor. Los autores mencionados critican algunos de estos estudios reseñados por sus graves errores metodológicos, no definir ni controlar adecuadamente las variables, y porque se apoyan más en procedimientos cualitativos de índole subjetiva y personal antes que en evaluaciones cuantitativas de naturaleza objetiva. Señalan que muchas de estas investigaciones se sustentan en el supuesto "aprendiz típico", el cual aprende únicamente por determinadas vías, no tomando en cuenta la gran cantidad de estilos cognitivos y la enorme diversidad de procesos de aprendizaje que utilizan los alumnos en la actualidad. 
Palloff y Pratt (2011), expertos en educación virtual, precisan que su propia experiencia con la enseñanzaaprendizaje "online" les ha permitido observar detalladamente la forma de comportarse de alumnos y docentes actuales en una clase presencial. Los actuales docentes ya no centran su labor en sus explicaciones orales presenciales, analizando y explicando a los alumnos los contenidos de los textos, Ahora, señalan estos autores, los alumnos están en condiciones de leer y comprender previamente los contenidos. Por tanto, la clase se convierte en un entorno para promover trabajos grupales de indagación y discusión de conocimientos, caracterizada por su naturaleza colaborativa y autónoma.

Según Miller y Miller (2012), luego de un análisis del futuro que le espera a la educación virtual, han logrado ubicar cuatro factores importantísimos que juegan un rol de primer orden en la conformación de este futuro: la permanente evaluación de su efectividad; la evaluación constante de los progresos científicos y tecnológicos; la medición de los costos de la competencia en el mercado educativo, y la respuesta de docentes y alumnos a las innovaciones de diferente índole propuestas por las autoridades educativas. De acuerdo con estos autores, son los resultados, junto con los aportes e innovaciones propuestas, derivados de estas investigaciones las que determinarán el futuro de la educación virtual como un sistema consolidado y exitoso de enseñanza - aprendizaje, a corto y mediano plazo. Es pertinente indicar que la mayoría de los estudios prospectivos acerca del futuro de la educación virtual reportan, en su gran mayoría, escenarios optimistas antes que escenarios pesimistas.

Beranguer (2017) en sus conclusiones acerca del aprendizaje inverso señala que las ventajas del método son innegables y su real eficacia funcional en las aulas ha quedado reiteradamente comprobada por numerosos estudios. La utilidad del aprendizaje inverso se incrementa en los casos de alumnos que se hallan en proceso de adaptación curricular. Este autor plantea que el éxito del modelo requiere de un alumno implicado al $100 \%$ dentro del proceso de enseñanza - aprendizaje, tipo de alumno difícil de captar actualmente. Por estas razones se señala que los beneficios del "aula invertida" no se generan solos. Al docente le corresponde motivar al alumno y promover -en otros docentes- el interés por este modelo de aprendizaje. Por esta razón, en primer lugar, se debe explicar a los alumnos los objetivos del aprendizaje, las metas finales, las exigencias y los procedimientos específicos, para -finalmente- lograr un trabajo eficiente y autónomo que sea permanente y se prolongue hasta el final de las tareas. Una estrategia adecuada puede ser recompensar a los alumnos que vayan logrando alcanzar progresivamente los objetivos mínimos planteados. Por ejemplo, otorgando puntajes adicionales a los que alcancen determinados promedios mínimos de rendimiento o de desempeño exitoso, tras ser sometidos a una capacitación específica. En este contexto, el objetivo primordial es lograr que el discente perciba y asuma la tarea de aprender no como una obligación forzosa sino, en términos de Einstein, que perciba y sienta la tarea de aprender como una posibilidad de ingresar y disfrutar del hermoso y apasionante mundo del conocimiento.

Merla (2018) acerca de la vinculación entre el aula invertida y el rendimiento académico manifiesta que la mayoría de las investigaciones planteadas para estimar cuantitativamente el impacto del aprendizaje inverso en el rendimiento académico informan la obtención permanente de resultados positivos en lo referido al aumento constante de desempeño académico de los alumnos. Estos logros altamente exitosos han despertado la curiosidad de los expertos por conocer mayores detalles del modelo aplicado, el tipo de estrategias didácticas, las técnicas y procedimientos, que posibilitan la aplicación del modelo. En este ambiente, el método del aprendizaje inverso es una alternativa a considerar ya que implica escoger un modelo comprobado de enlace exitoso entre las TIC y los procedimientos de instrucción estructurados en base a la ubicación e identificación de las necesidades de aprendizaje de los discentes. En este orden de ideas, Johnson et al. (2015) reseñan que el Report Horizont del año 2015, acerca de las nuevas modalidades educativas que repercutirán favorable y exitosamente en los futuros procesos educativos en los años venideros, ubica al modelo del aprendizaje inverso, que será -según sus vaticinios- cada vez más utilizado por las entidades de educación superior en los próximos años. 


\section{Justificación e importancia}

El presente estudio posibilitó, a través de un trabajo de campo, de naturaleza mixta (cuantitativa y cualitativa) y procesado estadísticamente, comprobar que el Programa de Educación Virtual basado en la modalidad de aprendizaje inverso, es una alternativa adecuada, eficaz y eficiente para promover los aprendizajes pertinente a la asignatura de Teoría de la Geografía en alumnos del tercero y cuarto año de la especialidad de Ciencias Histórico Sociales de la Facultad de Educación de la Universidad Nacional Federico Villarreal. 2021.

La importancia de este estudio se fundamenta en lo siguiente:

- El estudio de este tema es importante porque la actual pandemia del COVID 19 es un asunto de profundo interés local, regional, nacional e internacional, existiendo al respecto numerosas normativas nacionales e internacionales respecto a las modalidades educativas más apropiadas para abordar esta situación.

- Porque presentadas las conclusiones y recomendaciones del estudio realizado, entidades públicas y privadas pertenecientes al rubro educativo de la educación superior, podrán revisar el estudio y, de considerarlo pertinente, incorporar las sugerencias planteadas a fin de afrontar y superar los problemas y deficiencias encontradas y, por tanto, optimar los procesos educativos aplicados.

- Porque permitirá plantear pautas y procedimientos concretos dirigidos a afrontar y mejorar de los procesos educacionales en situaciones de emergencia sanitaria.

- Porque el presente estudio puede ser utilizado como referencia teórico - práctica y motivar su réplica con fines de corroboración de investigaciones similares en otros lugares.

\section{Marco teórico}

Beranguer (2017) acerca del aprendizaje inverso señala que el aula invertida es una modalidad instructiva cuya finalidad principal es que el estudiante se incorpore al proceso de aprendizaje desempeñando un papel mucho más activo que el que ha desempeñado habitualmente. En lo esencial, el modelo radica en que el discente aprenda los planteamientos teóricos por su propia cuenta recurriendo a variadas herramientas tradicionales y tecnológicas que el profesor debe proporcionarle, básicamente vídeos y multimedias explicativos y, que el valioso y escaso tiempo de clase sea utilizado positiva y constructivamente en la resolución de dudas y en la obtención de nuevo material relacionado con el tema o asignatura, aprovechando el tiempo disponible para realizar prácticas e intervenir en foros sobre temas controversiales.

En las actuales circunstancias, en que por razones de la pandemia Covid 19, los alumnos se han convertido en «e-alumnos», es decir, estudiantes que, tanto dentro como fuera del aula, utilizan las TIC como habituales herramientas de aprendizaje y que en el contexto digital es muy simple obtener nueva información, grabarla, editarla y difundirla, en suma acceder a materiales educativos, es de suma importancia estudiar la utilidad real de la Educación Virtual y del aprendizaje inverso en el nivel de la educación universitaria, así como de los diversos medios para aplicarlos y su efectividad real. Es un hecho que los estudios continuados y sistemáticos sobre este tema contribuirán a su perfeccionamiento y optimización.

Al respecto, un interesante estudio de Tourón y Santiago (2015) en Columbia (USA) demuestra que, de aproximadamente las doscientas palabras por minuto que puede pronunciar un docente, el alumno promedio sólo entiende el cincuenta por ciento; que los alumnos recuerdan aproximadamente sólo el setenta por ciento de lo expuesto en los primeros diez minutos de la clase y sólo retienen un $20 \%$ de lo expuesto en los diez últimos, y que permanecen en atención sólo el $40 \%$ del tiempo total que dura la clase. Por estas razones, las autoridades educativas y los docentes analizan con sumo interés cuál es el mejor procedimiento para transferir conocimientos y lograr que el discente incorpore efectivamente los contenidos educativos trasmitidos y obtenga el mayor rendimiento por las horas invertidas en su proceso de aprendizaje. En este contexto, el aprendizaje inverso es un sistema pedagógico que, bien aplicado, puede coadyuvar positivamente a lograr este propósito. 
González y Carrillo (2016) señalan que el aprendizaje inverso alude a un reciente modelo didáctico en el que los estudiantes aprenden los nuevos contenidos educativos a través de videos explicativos y demostrativos, habitualmente en su domicilio. Por esta razón, las tareas que antiguamente solían ser sus "deberes" para ser realizados en casa, ahora se realizan en el aula apoyados por el profesor el cual tiene, de esta manera, la oportunidad de proporcionar una orientación más precisa y personalizada, mediante su interacción directa y cooperativa con los alumnos.

El propósito fundamental de este modelo es estimular al estudiante para que trabaje individualmente y fuera del aula aquellos elementos teóricos y problemas, utilizando los diversos instrumentos que el docente pone a su disposición (vídeos, zoom, power point) y que la clase presencial se aplique preferencialmente para resolver interrogantes sobre el material proporcionado, así como para llevar a cabo prácticas educativo - grupales, plantear foros donde se discutan los temas educativos sugeridos o controversiales. Esto, como señalan Beesley y Apthorp (2010) es casi cuatro veces más efectivo que la práctica en la que los estudiantes ejercitan sus habilidades en clase apoyados únicamente con el background formativo y cultural del docente, con el agravante que, en este último caso, los docentes tienen mínimas oportunidades para supervisar. Con el nuevo método pedagógico, el alumno, si bien actúa de modo autónomo, nunca lo realiza aisladamente porque cuenta con la supervisión del docente mientras realiza el proceso de aprendizaje. El maestro desempeña su rol de apoyo y orientador asumiendo personalmente la tarea de seleccionar los contenidos a estudiar y analizar, poniendo a disposición del alumno -a través de diversos procedimientos- los recursos y medios apropiados para la adecuada asimilación cognitiva del alumno y permaneciendo en permanente contacto con él.

Esta nueva situación supone una inversión de roles con referencia al modelo tradicional porque con este procedimiento es el alumno quien interviene activa y directamente en su aprendizaje personal y el docente asume -por su parte- un rol de orientador y consejero.

\section{El método de aula invertida}

Los procedimientos que habitualmente se aplican en el aprendizaje inverso son:

1. El docente diseña, selecciona y planifica personalmente el proceso educativo concreto que se aplicará en una sesión específica, integrando estrategias, modelos de enseñanza y metodologías específicas.

2. El docente diseña y planifica las actividades específicas que los alumnos realizarán previamente fuera del aula (ver vídeos, analizar textos, llenar cuestionarios, realizar exposiciones, etc.).

3. El docente selecciona e integra la tecnología específica que se aplicará en las actividades de aprendizaje activo.

4. El docente entrega previamente a sus alumnos, utilizando procedimientos virtuales o presenciales, el material educativo referido a los contenidos educativos que se tratarán en clase.

5. Este material podrá ser presentado en diferentes modalidades: textos, videos, audios, presentaciones, mapas conceptuales, cuadros sinópticos, etc. que se tratarán en la siguiente clase o clases.

6. El docente diseña y explica a sus alumnos las actividades de evaluación específica que se aplicará para valorar el aprendizaje del alumnado.

7. El alumno disponiendo del material educativo, lo analiza y estudia -individual o grupalmenterecibiendo si lo considera necesario explicaciones complementarias por parte del docente y de ser el caso, accede a las actividades previas facilitadas por el profesorado.

8. Iniciada la clase, tanto docente como alumno, comparten ideas, resuelven interrogantes y resuelven dudas, en medio de un ambiente de colaboración y cooperación que estimula el pensamiento crítico y, sobre todo, creativo.

9. Finalmente, el docente procede a evaluar el aprendizaje de sus alumnos de acuerdo con los procedimientos explicados previamente. 


\section{El rendimiento académico}

Esta variable dependiente, en el caso del presente estudio, se conceptualiza habitualmente como el producto expresado cuantitativamente del nivel de la asimilación de los contenidos de aprendizaje. El desempeño académico es normalmente cuantificado mediante calificaciones dentro de una escala establecida. Es pues, el resultado cuantitativo que se obtiene en la medición de conocimientos incorporados, de acuerdo con evaluaciones que realiza el profesor a través de pruebas objetivas y otras actividades complementarias. El rendimiento académico expresa cuantitativa y objetivamente los niveles de conocimiento obtenidos consecuencia de la acción educativa aplicada. Se le considera como uno de los criterios principales para expresar el éxito o fracaso académico mediante un sistema de calificaciones de índole cuantitativa.

Según Bloom (2006) el rendimiento académico trasluce objetivamente el efecto de la acción educativa a lo largo de las diversas fases del proceso educacional. El rendimiento académico es la meta final hacia las que se orientan el conjunto de esfuerzos, iniciativas y decisiones de las autoridades educacionales, familias y comunidad en general. En resumen, el rendimiento educativo es el nivel de transformaciones cognitivas, afectivas y actitudinales operadas en el discente, a consecuencia del proceso de aprendizaje y que se expresa a través del crecimiento cognitivo, afectivo y actitudinal del alumno.

\section{Objetivos de la investigación Objetivo general}

Determinar si en la Educación Virtual la aplicación del método de aula invertida incrementa significativamente el aprendizaje de la asignatura Teoría de la Geografía en los alumnos del tercero y cuarto año de la especialidad de CC.HH.SS. de la Facultad de Educación de laUNFV. 2021

\section{Objetivos específicos}

Precisar si en la Educación Virtual la aplicación del método de aula invertida permite incrementar significativamente el aprendizaje de la asignatura de Teoría de la Geografía en la unidad I (Evolución histórica de la Geografía), II (Ciencia e investigación científica), III (Geografía cientíica), IV (Investigación geográfica)en los alumnos del tercero y cuarto año de la especialidaddeCiencias Histórico-Sociales delaFacultad deEducacióndelaUniversidad Nacional Federico Villarreal. 2021.

\section{Método y materiales}

El tipo de investigación fue "investigación tecnológica", también conocida como "investigación aplicada" ya que se propone aplicar y comprobar la efectividad de un programa para estimular el aprendizaje inverso en la asignatura de Teoría de la Geografía en alumnos del tercero y cuarto de la especialidad de Ciencias Histórico-Sociales de la Facultad de Educación de la Universidad Nacional Federico Villarreal. 2021. El estudio fue descriptivo comparativo por cuanto de describieron y compararon los desempeños de los grupos conformados (Experimental y de Control). El diseño de investigación asumido fue "cuasi experimental", específicamente el denominado "diseño antes y después con un grupo control no aleatorizado". Alarcón (2008) acerca de este diseño indica que es muy utilizado ya que es muy útil para determinar si se ha producido algún cambio en la conducta de los individuos entre su estado inicial, medido por el pretest, y la situación posterior, después de introducir el tratamiento". El diseño aplicado fueel "cuasi experimental" porque la conformación de los grupos fue no aleatoria porque se composición estuvo conformada en base a los criterios del investigador. El corte de la investigación fue longitudinal porque se desarrolló a lo largo de diversos intervalos de tiempo y presentó un enfoque cuantitativo porque sus resultados se expresaron en forma numérica y se procesaron estadísticamente.

\section{Población}

Estuvo constituida por la totalidad de alumnos que cursan estudios de Geografía en tercero y cuarto año de la especialidad de Ciencias Histórico-Sociales de la Facultad de Educación de la Universidad Nacional Federico Villarreal. 2021. La población, por tanto, estuvo constituida por 34 alumnos. 


\section{Muestra}

En consecuencia, la muestra de la investigación estuvo integrada por 34 alumnos, diecisiete (17) de los cuales formaron el Grupo Experimental y las otras diecisiete (17) restante integraron el Grupo Control.

\section{Instrumento}

La Prueba de Entrada y la Prueba de Salida. Fue un Cuestionario de Autoevaluación en formato tipo Likert. Abarcó las cuatro áreas temáticas del curso de Teoría de la Geografía. Consta de 16 ítems con cuatro alternativas de respuesta cada uno: 1) Conozco poco; 2) Conozco regular; 3) Conozco bien; 4) Conozco muy bien. Fue elaborado por los autores del estudio y cuenta con validez establecida por opinión de jueces (expertos) y confiabilidad establecida con la Prueba Alpha de Cronbach en una Prueba Piloto realizada previamente con 12 personas.

\section{Procesamiento estadístico}

A fin de contrastar las hipótesis planteadas se compararon los promedios obtenidos por los Grupos Experimental y Control en la Prueba de Entrada y Salida, por cuanto se aplicó un diseño estadístico de "comparación de medias". Como el pretest y el postest proporcionaron puntuaciones distribuidas de forma normal, la estadística a aplicarse fue la paramétrica. Se aplicó el estadístico más recomendable, tratándose de comparaciones de medias entre dos grupos, en este caso la Razón " $t$ " de Student.

\section{Resultados}

\section{Comprobación de la normalidad de la distribución de puntajes y de la igualdad de varianza}

Los grupos (Experimental y Control) presentaron una distribución normal, cumpliéndose así el primer requisito: Distribución normal de las puntuaciones de la muestra debidamente tabuladas.

\section{Prueba de Entrada}

a) Comparación Grupo Experimental y Grupo Control

Tal como se aprecia en el siguiente gráfico, las medias de los dos grupos en las diferentes áreas resultaron ser muy similares:

\section{Figura 1}

Puntuaciones en Prueba de Entrada. Grupo Experimental y Grupo Control.

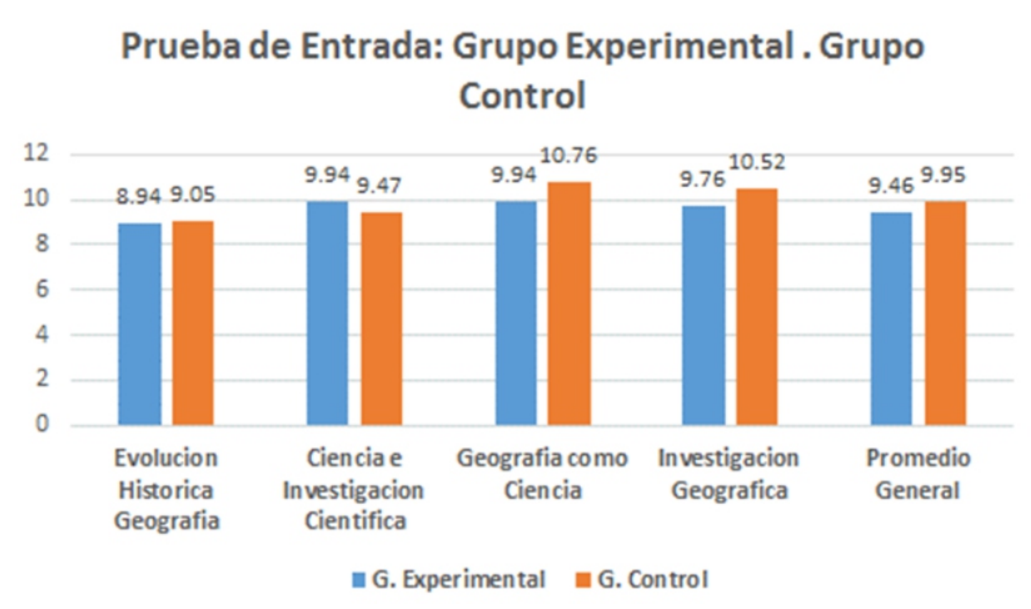

Los resultados significan que ambos grupos iniciaron la experiencia en similares condiciones.

\section{Prueba de Salida}

Los promedios de ambos grupos en la Prueba de Salida, difieren entre ellos, como se pueden apreciar en el siguiente gráfico: 


\section{Figura 2}

Puntuaciones en Prueba de Salida. Grupo Experimental y Grupo Control.

Prueba de Salida: Grupo Experimental. Grupo

Controll

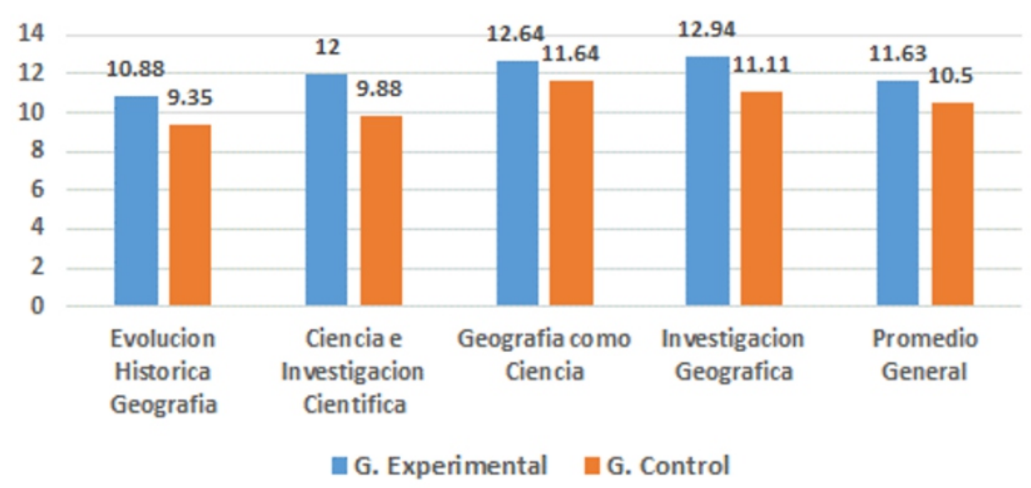

Se puede apreciar que en la Prueba de Salida ambos grupos presentan promedios diferenciados en sus diversas áreas.

Para comprobar si las diferencias encontradas en las diferentes áreas en la Prueba de Salida y si estas diferencias eran significativas estadísticamente, se aplicó la Razón " $t$ " de Student, con los siguientes resultados:

\section{Tabla 1}

Prueba " $t$ " de Student. Prueba de Salida

\begin{tabular}{lcc}
\hline \multicolumn{1}{c}{ Área } & $\begin{array}{c}\text { Razón } \\
\text { “t” }\end{array}$ & $\begin{array}{c}\text { Sig. } \\
\text { Bilateral }\end{array}$ \\
\hline Evolución Histórica de la Geografía & 2.346 & $\mathrm{p}=0.001$ \\
Ciencia e investigación Científica & 3.334 & $\mathrm{p}=0.001$ \\
Geografía como Ciencia & 1.870 & $\mathrm{p}=0.001$ \\
Investigación Geográfica & 2.784 & $\mathrm{p}=0.001$ \\
\hline Promedio General & $\mathbf{3 . 1 6 7}$ & $\mathbf{p}=\mathbf{0 . 0 0 1}$ \\
\hline
\end{tabular}

Se encontró que en todas las áreas, las diferencias entre los grupos Experimental y Control, presentaban una Significación Bilateral menor a $p=0.05$. Esto determinó que entre dichos grupos existían diferencias significativas en las diferentes áreas. Esto implica que ambos grupos iniciaros la experiencia en condiciones muy similares (Prueba de Entrada) pero terminaros la experiencia en diferentes condiciones. Al finalizar el estudio, el Grupo Experimental presentó en todas las áreas mejor rendimiento que el otro grupo, siendo estas diferencias estadísticamente significativas. La explicación de estas diferencias que favorecieron al Grupo Experimental debe ser atribuida -de acuerdo al diseño asumido-a la aplicación de la educación virtual (aprendizaje inverso) en contrastación con el Grupo Control, al que no se le aplico el indicado proceso.

\section{Discusión}

Los resultados del estudio coinciden con los de investigaciones similares sobre el tema, tanto a nivel local como en el exterior.

El estudio de Carbajal (2019) comprobó la eficacia de la enseñanza virtual en la modalidad del aprendizaje inverso indicando específicamente la necesidad que el docente se halle debidamente preparado para asumir las funciones que demanda esta modalidad formativa en el ambiente virtual. 
La investigación de Domínguez (2019) quien después de analizar las diferentes modalidades educativas, tanto presenciales como virtuales, concluyó que la moderna tecnología educativa puede favorecer su integración.

Palloff et al. (2011), analizando su experiencia con la educación virtual en sus diversas modalidades, incluida la de aprendizaje inverso, señalan que estas nuevas modalidades han cambiado radicalmente la forma como los alumnos actúan y conducen en una clase presencial tradicional, Ahora, ya no centran su trabajo en las exposiciones del docente quien asume la tarea de esclarecer los contenidos educativos expuestos sino que -actualmente- asumen ellos mismos la tarea de leer crítica y previamente el material educativo, elaboran sus propias exposiciones sobre los contenidos de los textos analizados, ejerciendo su actividad educativa de manera crítica y autónoma, dentro de un ambiente de colaboración y cooperación. En un ambiente así caracterizado, los alumnos deben desarrollar, -para adaptarse funcionalmente- variados mecanismos de autorregulación como: autodisciplina, autocontrol, autoaprendizaje, todo esto en un escenario caracterizado por un análisis crítico y reflexivo, potenciador de los intercambios cognitivos y vivenciales.

Miller et al. (2012) pronostican la futura consolidación de la enseñanza virtual y del aprendizaje inverso ya que los constantes desarrollos tecnológicos en equipos y programas ofrecerán cada vez más eficaces herramientas para la educación virtual. Señalan que diversos especialistas auguran que este tipo de educación será objeto de permanentes actualizaciones para responder adecuadamente a las cada vez más frecuentes innovaciones tecnológicas, convirtiéndose a mediano plazo en una cada vez más importante opción académica a considerar.

Beranguer (2017) en sus conclusiones acerca del aprendizaje inverso señala que sus beneficios son indiscutibles y que su eficacia y eficiencia, han quedado reiteradamente verificada a lo largo de numerosas investigaciones. Indica que en un futuro cercano la educación virtual se convertirá en un recurso al que habrá que recurrir para hacer frente a los impostergables cambios de diferente tipo que se avecinan a consecuencia de la evolución social y tecnológica mundial. Una de las razones de su desarrollo es que la virtualidad educativa permite al estudiante compartir sus experiencias educativas de manera directa con sus docentes y con sus compañeros de aula, reforzándose de este modo la cohesión y el trabajo en grupo.

Merla et al. (2018) acerca del tema de la presente investigación señala que los diversos estudios consultados en este sentido comunican resultados beneficiosos en lo que atañe al aumento significativo de los promedios de rendimiento académico en los estudiantes expuestos a esta modalidad educativa, lo cual ha generado interés en los expertos por ubicar, identificar conocer y optimizar las estrategias didácticas asociadas. Señala que el aprendizaje inverso es una modalidad educativa sustentada en una combinación eficaz entre la tecnología educativa y los procesos instruccionales estructurados en la previa evaluación y determinación de las insuficiencias y limitaciones detectadas en los alumnos. Vaticina que este enfoque será cada vez más aplicado y utilizado por las instituciones universitarias, tanto a nivel local como internacional, en base a su éxito y funcionalidad reiteradamente constatadas. Esta autora, indica que docentes, alumnos, directivos, autoridades educativas estatales y la comunidad en general deben introyectar una cultura del cambio educativo sustentada sólidamente en lo doctrinario, en lo conceptual y en lo operativo. Deben ser conscientes que la virtualidad implicará un nuevo modo de convivencia y asimilar vivencias educativas, el uso generalizado de la Web, y cambios en los sistemas vida tradicionales, situaciones éstas que impelen a asumir la modalidad educativa virtual sea cada vez más urgente. Precisa que los docentes, en su gran mayoría necesitan cambiar su actitud y disposición para afrontar este nuevo reto, pues es difícil tener éxito con una propuesta formativa virtual, si los directivos y profesores no están convencidos de la calidad y efectividad de estas modalidades mediatizadas por la virtualidad. 


\section{Conclusiones}

- En términos generales, la investigación corrobora la relevancia de la aplicación de la metodología del aprendizaje inverso para incrementar el desempeño académico de alumnos de nivel universitario en la asignatura de geografía.

- Se constató la Hipótesis General alterna que señalaba que, si se aplicaba el método del aprendizaje inverso en la asignatura de Teoría de la Geografía, a consecuencia de esta aplicación, el Grupo Experimental presentaría un mejor rendimiento que el Grupo Control en el desempeño en esta asignatura.

- Se comprobaron las Hipótesis Específicas que planteaban que la aplicación del método del aula invertida en las asignaturas de Teoría de la Geografía (Unidad I: Evolución histórica de la Geografía), Unidad II: Ciencia e investigación científica), (Unidad III: Geografía como ciencia), (Unidad IV: Investigación geográfica), al Grupo Experimental, generaría que este grupo evidenciara un mejor rendimiento que el Grupo Control en los aprendizajes de estas asignaturas.

\section{Referencias}

Alarcón, R. (2008). Métodos y diseños en la investigación del comportamiento. Lima: UPCH.

Barrenechea M. (2020). La gran prueba de la educación virtual: ¿Cómo un docente puede entusiasmar a sus estudiantes? Lima. RPP. https://rpp.pe/peru/actualidad/coronavirus-en-peru-la-gran-prueba-de-la-educacionvirtual.

Beesley, A. E. \& Apthorp, H. S. (2010). Classroom Instruction That Works. Second Edition: Research Report, Ed. Midcontinent Research for Education and Learning.

Beranguer, C. (2017). Acerca de la utilidad del aula invertida o flippedclassroom. España. Universidad Alicante.

Bloom, B. S. (2006). Bases Psicológicas de la Educación. México: Editorial Interamericana.

Cantoni, M. (2018). Posibilidades de innovación en educación a distancia en el Perú a través de los recursos educativos abiertos. Lima. Universidad Inca Garcilaso de la Vega.

Carbajal, G. (2019). Competencias profesionales del docente en un entorno virtual de aprendizaje. Propuesta normalizada basada en el análisis funcional. Lima. Universidad Católica Sedes Sapientiae (UCSS).

Domínguez, J. (2019). Hacia la convergencia de las modalidades educativas. Chimbote. Red de Universidades Inclusivas de Virtual Educa (RUIVE). Universidad Católica Los Ángeles de Chimbote.

González, N. \& Carrillo G. A. (2016). El Aprendizaje Cooperativo y la Flipped Classroom: una pareja ideal mediada por las TIC. Aularia: Revista Digital de Comunicación, 5 (2).

https://dialnet.unirioja.es/servlet/articulo?codigo $=5423145$

Merla, A. \& Yáñez, C. (2018). El aula invertida como estrategia para la mejora del rendimiento académico. Revista Mexicana de Bachillerato a Distancia, 8 (16). http://revistas.unam.mx/index.php/rmbd/article/view/57108

Miller, S. M. \& Miller, K. L. (2012). Theoretical and practical considerations in the design of Web based instruction. Beverly Abbey (Ed.) Instructional and Cognitive Impacts of Web-Based Education. Hershey, PA: Idea Group Publishing.

Muñoz, L. (2020). COVID-19: Detalles sobre la enseñanza a distancia. Lima. Facultad de Educación. Universidad Peruana Cayetano Heredia (UPCH).

Palloff , R. M. \& Pratt, K. (2011). The Virtual Student. A Profile and Guide to Working with Online Learners. San Francisco, EE.UU: Jossey-Bass.

Phipps, R. \& Merisotis, J. (2009). What's the difference? Washington, D.C.: Institute for Higher Education Policy.

Rodríguez, J. (2019) Aproximaciones a la educación a distancia en el Perú. Virtual Educa. Observatorio de la Educación Virtual de América Latina y el Caribe. Universidad de la Empresa. Montevideo. Uruguay.

Tourón, J. \& Santiago, R. (2015). El modelo Flipped Learning y el desarrollo del talento en la escuela. Revista de Educación, 368. https://dialnet.unirioja.es/servlet/articulo?codigo=5028544 


\section{Cómo citar este trabajo}

Alva Miguel, W. H., Matos Huaman, C., Zúñiga Reynoso, C., \& Wong Fajardo, E. M. (2021). Eficacia del método del aula invertida en la educación virtual en estudiantes universitarios. EDUCARE ET COMUNICARE: Revista De investigación De La Facultad De Humanidades, 9(2), 17-29. https://doi.org/10.35383/educare.v9i2.676

\section{Financiación}

El presente artículo no cuenta con financiación específica para su desarrollo y/o publicación.

\section{Conflicto de interés}

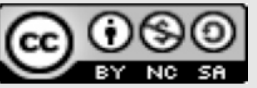

(c) Los autores. Este artículo es publicado por la Revista Educare et Comunicare de la Facultad de Humanidades, Universidad Católica Santo Toribio de Mogrovejo.

Este es un artículo de acceso abierto, distribuido bajo los términos de la Licencia Creative Commons Atribución-NoComercial-CompartirIgual 4.0 Internacional (CC BY- NC-SA 4.0), que permite el uso no comercial, distribución y reproducción en cualquier medio, siempre que la obra original sea debidamente citada. 